\title{
LES OBJETS-SIGNES CHEZ NINO RICCI ET BIANCA ZAGOLIN
}

\author{
ANNE MARIE MIRAGLIA
}

\begin{abstract}
Resumé: Dans un nouveau pays, la présence d'objets familiers, dotés d'un sens spécial, réconfortent l'immigré et contribuent à la reconstruction dans son pays d'accueil d'un monde révolu et lointain. Cette analyse de deux romanciers canadiens d'origine italienne, Nino Ricci (Ontario) et Bianca Zagolin (Québec), examine la nature et la signification des objets ainsi que leur rôle dans la caractérisation des personnages et dans l'architecture des textes. Les objets emportés par les immigrés de Ricci et de Zagolin servent de support à une mémoire faillible : ils représentent à la fois tout ce que l'immigré a perdu et tout ce qu'il essaie de retenir. Témoins d'un autre monde, les objets évoquent simultanément leur matérialité et quelque chose d'autre qui les dépasse.
\end{abstract}

Nino Ricci est né à Leamington, en Ontario, en 1959 de parents immigrés originaires du Molise, dans le Sud de l'Italie tandis que Bianca Zagolin est originaire d'Ampezzo, au Frioul, en Italie septentrionale. À l'âge de neuf ans, elle émigre avec sa famille au Québec où elle adopte le français comme langue d'expression. Ces distinctions biographiques et linguistiques se retrouvent au niveau des personnages dans la trilogie de Nino Ricci, qui comprend Lives of the Saints (1990), In a Glass House (1993) et Where She Has Gone (1997) et, dans les romans de Bianca Zagolin, Une femme à la fenêtre (1988) et Les Nomades (2001). Les lecteurs renseignés sur les difficultés sociales qui continuent de diviser l'Italie du Nord industrialisée et l'Italie du Sud pauvre et marginalisée remarqueront le transfert de ces différences sociales et régionales dans la fiction des Italo-canadiens.

L'histoire de Vittorio Innocente débute à Valle del Sole, dans le Sud d'Italie, un jour de juillet 1960 lorsque le garçon n’a que sept ans. La narration de cette histoire est prise en charge par Vittorio lui-même, le narrateur autodiégétique du récit. La focalisation du récit se limite au point de vue de l'enfant dans Lives of the Saints mais suivra le mûrissement du narrateur-protagoniste dans les volumes subséquents. L'histoire se termine vingt-et-un ans plus tard après un bref retour au pays natal.

Dans In a Glass House, il est évident que le drame familial occasionné par l'infidélité de Cristina dans Lives of the Saints, est symptomatique du 
drame collectif provoqué par les conditions socio-économiques et vécu par les paysans italiens contraints de quitter leur femme et leurs enfants pour chercher du travail à l'étranger. Dans Lives of the Saints, la crainte de l'infidélité de leur épouse inspire de la méfiance voire une certaine misogynie chez les hommes : "Their fears had given birth to a wealth of proverbs : 'guard your women like your chickens,' they said, 'or they'll make food for the neighbour's table' " (p.140). Les femmes et les enfants abandonnés, les maisons vides et en ruine et les villages en voie de disparition, prédominants dans le premier roman de Ricci (p. 85), se trouvent aussi dans Avril ou l'antipassion (1990) et dans Le Figuier enchanté (1992) dont les auteurs Antonio D'Alfonso et Marco Micone sont, comme Ricci, originaires du Molise.

Le premier et le deuxième roman de Bianca Zagolin sont relatés à la troisième personne par un narrateur qui privilégie la perspective d'Aurore, une veuve bourgeoise dans Une femme à la fenêtre, et le point de vue de sa fille, Adalie, et de Philippe dans Les Nomades. Philippe est un jeune étranger polyglotte de noblesse anglaise par son défunt père et française par sa mère. Adalie, dont la mère s'est suicidée, termine son internat dans un hôpital psychiatrique.

À part la description de familles brisées, d'enfants orphelins et de protagonistes exilés dont seules deux, Aurore et Adalie, sont d'origine italienne, les textes de Zagolin, dont l'intrigue se déroule en français, à Montréal, ne semblent pas, à première vue, partager de points communs avec ceux de Nino Ricci, centrés sur le milieu rural et italien de Valle del Sole, de Mersea en Ontario et finalement urbain et anglais de Toronto. Si les textes de Zagolin se distinguent de ceux de Ricci et des autres romanciers italo-canadiens du Québec c'est en partie parce que les personnages de Zagolin n'appartiennent pas à la masse de misérables qui ont quitté les terres féodales du Sud de l'Italieafin de trouver des emplois dans les villes canadiennes. Les Italiennes de Zagolin, Aurore et Adalie, appartiennent "à une classe privilégiée, la catégorie de ceux qui ont des parents haut placés. [Aurore] n'irait donc pas grossir les rangs des démunis en transfert "(Une fermme à la fenêtre, 49). Parallèlement, dans Les Nomades, pour rassurer ses petits camarades bourgeois qui ont vu la misère italienne dans les films Miracle à Milan et dans Le Voleur de bicyclettes, la petite Adalie leur confie: "Chez nous, c'était très beau. Ma chambre s'ouvrait sur une terrasse à balustres et on pouvait cueillir des prunes et des kakis dans notre jardin " (Les Nomades,127). Avant d'émigrer, Aurore et ses filles habitaient une petite ville du nord de l'Italie; elles étaient sous la tutelle de son mari médecin et, à la mort de celui-ci, de son frère ambassadeur vivant au Canada. 
Contrairement à ses compatriotes démunis du Sud de l'Italie remplis d'espoir à l'idée de réaliser leur rêve américain, Aurore ne voit dans son départ que le germe d'une aventure et la possibilité d'une certaine liberté. Aurore, elle, n'est pas riche, mais elle ne verra jamais l'intérieur d'une usine. Tout en restant lucide, elle entrevoit dans l'idée de l'Amérique la possibilité d'une liberté, troublante certes, mais tout à fait inconcevable en Italie :

La voix d'outre-mer, Aurore le savait, cachait dans ses tons mielleux tous les déchirements à venir : l'abandon d'une patrie ensoleillée pour un pays de neiges et de glaces, d'une terre parfumée où la mort elle-même dans ses plus éclatantes figures, vous attache à la vie; l'effritement des certitudes quotidiennes jamais remises en question, celles d'un mode de vie qui, tant bien que mal, a fait ses preuves, d'une langue qui offre ses ressources sans hésitation, même dans le silence des pensées les plus intimes; la fin de la possession tranquille de son espace vital, si restreint fût-il. Mais, là-bas, on se souciait moins de valeurs sûres, et puis c'était très grand, répétait l'oracle, et plein de chances d'avenir. Grand à s'y perdre, s'inquiétait Aurore. (p. 28)

Compte tenu de ces différences régionales, sociales et économiques caractérisant le milieu fictif de Ricci et de Zagolin, nous croyons qu'il serait intéressant de nous attarder sur la nature et la signification des objets que les exilés de Zagolin et Ricci emportent avec eux, sur le rôle des objets non seulement dans la caractérisation des personnages et dans l'architecture des textes mais aussi dans l'expression de certaines idées sur le passé et sur la mémoire.

\section{Les objets chez Nino Ricci}

Issus d'un milieu très pauvre, il est normal que les personnages de Ricci possèdent très peu de choses et que celles qu'ils emportent en Amérique n'aient de valeur autre que pratique ou affective. Les affaires de Cristina et du petit Vittorio entrent dans une seule malle en quelques heures et la maison ressemble, en un soir, à une coquille vide (p.164). Dans son désir de rupture d'avec les villageois, hostiles à son égard, Cristina, enceinte d'un enfant illégitime, n’accepte pas de transporter des paquets à leurs parents exilés. Aucune de ses possessions personnelles n'est identifiée. Mais son fils, le petit Vittorio, emporte quelques cadeaux qui demeureront précieux pour lui bien longtemps après leur perte matérielle. Il en est ainsi du couteau de poche que lui donne le petit Fabrizio, du livre Lives of the Saints que lui offre la maestra, des médailles de guerre de son grand-père et la pièce d'une lira que lui donne Luciano comme porte-bonheur. Le couteau, le livre, les médailles, la pièce - chacun de ces objets dotés d'une valeur affective 
trouvera sa place dans les deux volumes subséquents de Nino Ricci et contribuera à la cohérence structurale de la trilogie en plus de soutenir une mémoire faillible et floue. Dans In a Glass House, le livre de la maestra, sera remplacé par The Guiding Light dont les histoires semblent à Vittorio plus importantes car elles proviennent de la Bible et sont écrites en anglais (p.56). Vittorio perdra les médailles de son grand-père qui mourra, le pauvre, sans jamais le revoir. Mais cet ancien maire de Valle del Sole léguera à son petit-fils la maison familiale qui facilitera son retour à Valle del Sole vers la fin de Where She Has Gone. Aussi l'évocation du couteau de poche suffira-t-elle pour renouer l'amitié entre Vittorio et Fabrizio vingt ans plus tard.

Il est évident que ces cadeaux emportés dans l'exil prolongent les liens affectifs dans le temps et dans l'espace et font surgir constamment à la mémoire les qualités humaines de ceux qui ont marqué l'enfance du jeune garçon: le courage et l'altruisme du grand-père, l'affection protectrice de la maestra, l'amitié sincère et fidèle de Fabrizio. Parmi les objets que Vittorio emporte avec lui sur le paquebot à destination de l'Amérique, le plus significatif sur le plan symbolique est sans aucun doute la pièce d'une lire perdue dans l'Atlantique le soir de la grande tempête qui emporta la mère de Vittorio. Or cette pièce perdue sera remplacée à Mersea par une pièce tout à fait identique, fabriquée la même année, avec la même brèche minuscule sur l'aile de l'aigle (In a Glass House, 282). C'est un vieil Italien interviewé par Vittorio au cours de ses recherches sur les Italiens de Mersea qui lui donnera cette pièce. Et l'existence de cette pièce, identique à la première, ébranle sa conception du temps et provoque la première d'une série de remises en question sur la justesse des " réalités " que Vittorio a emportées. avec lui au Canada.

A glint of silver at the bottom of the box caught my eye- a coin, an old one lira, I recognized it at once. I reached for it and a thrill passed through me like a premonition: the coin was the same as the one I'd been given years before by my morher's friend Luciano, the same year, 1927, the same nick in the eagle's wing that he'd said had stopped a bullet from entering his heart during the war. For an instant time seemed to falter and to warp. (In a Glass House, 282-283)

A la veille de son retour en Italie, les objets que le petit Vittorio avait emportés avec lui en Amérique ont disparu depuis longtemps déjà. Cette deuxième pièce, identique à la première, parvient à représenter tout ce qu'il a perdu et, de façon générale, tout ce qu’on essaie de retenir, de garder en souvenir au moyen d'objets dotés d'une valeur symbolique mais essentiellement illusoires et inutiles pour la reproduction du réel : 
The coin seemed now the symbol of everything that had vanished from my life, in every respect the same as the first except in the important thing of being the actual physical thing, what had passed from hand to hand, what could have proved the reality of a certain moment or person or place. It hardly seemed possible sometimes that a life could go on at all with only such phantoms to lend it credence, with almost nothing that could ever be nailed down for certain. Yet that was what matters always came down to, to faulty recreations of things that had themselves perhaps only been tokens, that hadn't been adequate even in their first moment of meaning to take in the fullness of the world that they'd strained to represent. (Where She Has Gone, 159)

C'est cette deuxième pièce que Vittorio offre à Fabrizio lorsqu'il retourne à Valle del Sole et, par ce geste, il répare finalement un manque vieux de vingt ans qui n'a cessé de le miner.

Il est évident dans le passage ci-dessus que la représentation des objets chez Ricci dépasse la simple fonction descriptive, ce que Roland Barthes a appelé l'" effet de réel ". Les objets sont chez Ricci dotés d'une valeur symbolique et cela souvent au niveau de la fiction par les personnages euxmêmes. Comme le signe linguistique saussurien, les objets de Ricci comportent une dimension "signifiant " ou matérielle, et une dimension " signifié " ou conceptuelle. Semblables aux mots tracés par Vittorio isolé dans une île du Kenya à la fin de Where She Has Gone, d'où il essaie de représenter le monde de Valle del Sole, de Mersea et de Toronto, les objets dans la trilogie de Ricci représentent quelque chose d'autre qu'eux-mêmes, en d'autres termes aliquid stat pro aliquo. Or, en plus d'évoquer les absents et leurs qualités humaines, le couteau de poche, le livre de la maestra, les médailles du grand-père et la pièce d'une lira renvoient Vittorio à ses insuffisances personnelles et à son habituel sentiment de perte et de non appartenance.

Le narrateur de Ricci cherche constamment dans les objets des signes susceptibles de dévoiler le mystère entourant les êtres humains, mais souvent ces mêmes objets ne reflètent que sa propre aliénation. Par exemple, quand le perit garçon arrive pour la première fois dans la maison de son père, Vittorio remarque que Mario possède si peu d'affaires personnelles qu'il est impossible de deviner la durée de son séjour sur la ferme. L'étrangeté de ce nouveau monde se répercute aussi dans les meubles d'occasion qui, semblables à leur nouveau propriétaire, taciturne et sombre, suggèrent leur altérité tout en refusant de livrer leur histoire :

But if the house had any informing spirit it didn't seem to reside in its objects, which despite their novelty gave no feeling of welcome to the 
rooms that held them, refusing to give up their histories, sitting stubborn and mute in their separate spaces like things that had turned their backs to you. The only thing that betrayed them in this was a smell, [...] lingering on the furniture, in the cupboards in the kitchen and pantry, in the chintz curtains left at the windows, and stealing over me sometimes to leave an odd hollowness in me like a gloom, the creeping intimation of whatever unknown lives had gone on there before us. (p. 6)

Ainsi, dans cette maison repliée sur elle-même où chaque objet suggère quelque chose d'autre qui le dépasse, même la petite demi-sœur de Vittorio acquiert le statut de signe rappelant à Mario et à Vittorio leur honte et leur humiliation. Dans la maison, s'insinue progressivement une division spatiale et psychologique dont l'objectif primordial est de faire oublier autant que possible l'existence de la petite. Cette démarcation est cependant troublée à l'achat d'une télévision qui deviendra le lieu de résistance pour la petite Rita. En plus de propulser la famille dans la modernité (p. 88), cette nouvelle acquisition suggère la possibilité de ce que Vittorio appelle " normalcy " (p. 92) pour sa famille. Et avec le changement de l'heure du dîner occasionné par l'émission "Lassie ", Vittorio remarque " some shedding of our habitual immigrant unrefinement » (p. 92).

Il est évident que le sentiment d'humiliation, traduit ci-dessus par l'attribut " immigrant ", dépasse largement des considérations d'ordre économique. Si le nombre progressivement croissant des serres construites sur la ferme atteste ponctuellement le succès et la richesse grandissants de la famille, la mentalité, les habitudes et les manières apprises dans le milieu démuni de Valle del Sole demeurent bien longtemps après l'arrivée de la fortune. Ainsi, rien dans les habits, dans les habitudes, dans le comportement de Mario et des siens ne signale la somme exorbitante de la fortune que ce père léguera à son fils.

Yer it was odd when we'd so clearly prospered that my memory of our years on the farm till then was only of setbacks, the constant sense that we verged always on the brink of ruin; [...] living our lives with the same frugality, the same sense of threat, as the peasants in Italy who'd wondered from one year to the next if their harvest would last the winter. When I thought of us there on the farm, it was always with this feeling not so much of having moved forward as of having struggled to remain the same, forever stranded as on an island within the tight logic and rules of what was acceptable, how far we could reach. (In a Glass House, 190)

D’ailleurs, Mario, ce personnage sombre et coléreux, quitte ce monde en laissant certes une ferme prospère mais très peu d'objets personnels : 
quelques vêtements, un rasoir électrique, une chaîne en or avec une croix et deux anneaux, dont son alliance. Et quoique son héritage permette à Vittorio de vivre longtemps comme il le veut sans soucis financiers, le jeune homme vit à Toronto avec la même frugalité que son père dans un appartement meublé d'occasion. Vittorio, cependant, n'a pas envie d'endosser la vie de Mario. En vendant la ferme à son oncle et à ses cousins, Vittorio rompt de façon définitive avec la vie de son père. Cette rupture, il l'avait déjà prévue, plusieurs années plus tôt, lorsque l'inscription "Innocente Farms Ltd " avait remplacé " Mario Innocente \& Son " sur le vieux camion de son père (p. 189).

Semblables au décor sur une scène de théâtre, les objets évoquent pour Vittorio simultanément leur matérialité et autre chose. Cet " autre chose» prend souvent la forme d'un sentiment, signifiant, à son tour, la façon dont Vittorio conçoit ses rapports avec le monde. Comme exemple, examinons l'impact des lettres de Cristina que Vittorio découvre un jour par hasard, inséparables de leurs enveloppes, endommagées par l'eau d'un incendie. La tante Teresa jettera cette masse collée avant que Vittorio ne puisse les lire. Or la perte de ces lettres suscite chez Vittorio un soulagement coupable et l'impression que quelque chose de crucial lui manque :

But my search for them lacked conviction: I felt only the guilty relief of being free of them of their uncertain burden, was left again with the sense that something crucial was missing in me, normal human emotion, the way I reacted to things with only this emptiness at the centre of me (In a Glass House, 129)

Ce sentiment de vide, cependant, n'empêche pas Vittorio d'être sensible ni à la présence de son père lorsqu'il prend possession de la voiture du défunt ni à la sensation d'effacer sa trace en changeant la disposition du rétroviseur et du siège. Il est aussi significatif que Vittorio ne ramasse pas les objets dans la voiture de son père - un paquet de cigarettes, un programme d'église - qui rendent presque tangible la présence du défunt dans le siège du passager. Ainsi le comportement de Vittorio par rapport aux objets révèle des tensions personnelles le tiraillant entre le désir de rapprochement et le besoin d'éloignement face à un père dont l'ombre menace de l'engloutir.

Que l'importance fondamentale des objets réside chez Ricci essentiellement dans ce qu'ils révèlent sur la nature humaine et sur les rapports de l'individu avec le monde est aussi évidente dans la façon dont Mme Amherst entretient sa maison: "Around her the house sat in calm, quiet orderliness like an extension of her: this was what she had always been to 
me, these varnished tabletops, these lace doilies, this cut glass; these were the things that had always seemed to hold her in place" (Where She Has Gone, 34). Comme Rita et Elena, l'autre fille adoptive des Amherst, Vittorio est lui aussi troublé par le projet de Mme Amherst de rentrer en Angleterre à la mort de son mari : "There seemed something unconscionable in this, preposterous, that these solidities she had ruled over could be allowed to vanish without a trace " (p. 35). Et Vittorio comprend qu'avec le départ de Mme Amherst, la vente de la maison, la disparition de ses objets " the very foundations of Rita's and Elena's lives, their whole idea of home, had shifted, fallen away " (p. 35). Si l'œuvre de Ricci évoque la notion de "home " (d'un chez-soi) c'est souvent, comme dans le passage ci-dessus, dans le contexte de son absence. Et cela parce que Vittorio n'est " chez lui " nulle part, ni à Valle del Sole, ni à Mersea, ni à Toronto. Le concept de " home " dépasse largement toute manifestation matérielle: les objets ne peuvent que le suggérer. Tout comme ils ne peuvent que suggérer la présence des êtres humains sans les reproduire.

Aussi la limite des objets se précise-t-elle lorsque Vittorio s’insinue par un subterfuge dans l'appartement de John, l'homme qu'il soupçonne être le père de Rita. Les objets dans l'appartement révèlent entre autres que cet homme aux yeux bleus, comme ceux de Rita, est d'origine allemande, est arrivé au Canada, comme Vittorio, au début des années 60 , a obtenu la citoyenneté canadienne, est peut-être professeur et lit beaucoup de livres notamment sur l'Holocauste. Mais aucun objet ne révèle à Vittorio ce qu'il cherche à savoir. Les objets sont incapables de dévoiler tous les secrets d'un individu, il manque toujours l'essentiel.

Et Vittorio de reconnaître que les choses dont on s'entoure sont semblables au détritus accumulés contre une palissade (" the generic debris that accumulated against us like litter against fences "Where She Has Gone, 145). Les objets qui nous entourent ne suffisent ni à faire un " home " ni à révéler l'identité profonde des êtres. Les objets sont signifiants, mais ne signifient que dans la mesure où l'être humain leur donne un sens. Cela devient plus clair lorsque Vittorio visite un musée italien accompagné de Rita :

[...] the curator explained how much could be learned, deduced, across the millennia from these few shreds of things, as if everything of importance had left its indelible trace. I thought of my stumbling attempts to get at the truth of my own past: it seemed that more could be known for certain about these ancient ancestors, about events that had taken place at an unfathomable distance of years, than of what by comparison had happened hardly a moment ago. (Where She Has Gone, 263)

Il n'est pas surprenant que Vittorio abandonne son appartement, 
vende ses meubles et renonce à ses possessions pour partir en Italie avec seulement deux valises, les lettres de Rita, The Guiding Light et les rares objets appartenant à son père.

I came from a line, it seemed, that did not hold on to things, that had no heirlooms to pass on, no signet rings, that didn't think itself a weighty enough presence in the world to leave some record of its having passed through. I thought of the gifts I'd brought with me from Italy, a jackknife, a Lives of the Saints, my grandfather's war medals, things that I might have passed on to a daughter or son along with their stories but all scattered now, lost like memories I could not quite recall. (p. 159).

Or une fois de retour à Valle del Sole, tout ce qui a été abandonné vingt ans plus tôt, tout ce qui a perdu pour Vittorio au Canada un semblant d'existence - son village natal, la maison familiale, le paysage de ses jeux d'enfants - tout ce monde est réinvesti d'une réalité qui n’est chimérique que considérée de loin. (p. 155). Et quoique la présence matérielle des choses à Valle del Sole serve de preuve contre tout soupçon d'une existence rêvée ou imaginée, stimule la mémoire et donne une forme plus réelle aux gens et aux événements du passé, Vittorio s'aperçoit que rien n’est plus comme avant, et qu'on ne peut revenir en arrière : "Everything was as I remembered it and not, was familiar in some wordless, visceral way and yet utterly foreign and shrunken, too tangible somehow to be real "(Where She Has Gone, 183). La simplicité du village frappe Vittorio et suggère l'improbabilité d'un quelconque événement remarquable (p. 178). Parallèlement, la simplicité grave de la jeune montagnarde qu'il voit dans la photo prise, peut-être, le jour de leur départ cloche avec l'image qu'il a gardée d'une mère belle, dynamique et souriante. De plus, chacun de ses souvenirs - la pluie le jour de son départ, l'absence d'électricité dans le village, son lâche abandon de Fabrizio - tout le passé construit par le garçon de sept ans est nié par des versions multiples d'un passé foncièrement insaisissable :

I had begun to forget things. Memories that had seemed clear when I first arrived were becoming more and more contaminated, overlaid by other people's versions of the past or simply by mere reality, the different slope of a street [...] I'd hear some new story or fact and at once all the careful architecture of the past that I'd carried around in my head seemed to shift to make a place for it, my brain producing images that might have been memories or pure inventions, just the mind's attempt to connect things. [...] It was as if I'd come here not to remember but merely to put together a plausible story: these were the elements, I was free to arrange them how I wished. (Where She Has Gone, 230-231) 
L'impossibilité de réconcilier le passé avec ses souvenirs, l'inconsolable sentiment de perte, de dislocation et sans doute un certain mépris pour luimême occasionné par l'inceste avec Rita aggravent la dépression de Vittorio qui abandonne à Rome le peu qui lui reste de ses affaires avant de prendre une chambre dans un hôtel minable de Londres où il se coupera les veines pour se délivrer d'un dernier objet, d'un dernier poids, celui insupportable de son propre corps.

Sauvé malgré lui, Vittorio s’isole sur une île du Kenya à partir de laquelle il écrit son histoire, cherche avec des mots à ressusciter Valle del Sole et sa mère Cristina, Mersea et son père Mario et à réfléchir sur ses relations futures avec sa demi-soeur. Quoique Vittorio condamne l'inceste, se considère monstrueux, le texte semble suggérer qu'il est logique et peut-être même nécessaire que les relations de ces deux orphelins arrivent à ce point dramatique avant que chacun d'eux puisse trouver le moyen de s'extirper l'un de l'autre pour devenir deux corps, je dirais même deux objets, n'appartenant à personne et trouvant chacun en lui-même la signification de son existence.

'Sometimes I feel like I've never had anything,' she said. 'Anything that was really mine.'

'I know what that's like. I suppose I always thought that you were what was mine'

'How do you mean?'

'I don't know. Just that. That I wasn't anything, really, except for you. I guess I hated you for that in some ways.' (p. 69).

En plus de ramener Vittorio à lui-même, l'écriture, comme la pièce d'une lire, donne une forme et un sens à des choses disparues depuis longtemps.

In the meantime I set things down, placing them one after another like links in a chain that might finally pull me back to the world, though there remain always those things, perhaps the most important ones, that are not quite captured or that are held back, where ability fails or where every fibre rebels at the betrayal of putting a thing into words. Language seems sometimes such a crude tool to have devised, obscuring as much as it reveals, as if we are not much further along than those half-humans of a million years ago with their fires and their bits of chipped stone; though maybe like them all we strive for in the end is simply to find our own way to hold back for a time the encroaching dark. (p. 320)

Ainsi, l'ampleur de l'intertexte proustien qui sert d'exergue à Lives of the Saints n'est évidente que dans le dernier volume de la trilogie. Comme Marcel, le protagoniste-narrateur qui fait surgir d'une madeleine l'univers 
fictif d'À la recherché du temps perdu, Vittorio se sert des objets, comme des mots, pour soulever des questions se rapportant à la fuite du temps, aux défaillances de la mémoire et à la difficulté, voire à l'impossibilité de représenter le réel à travers la langue et l'écriture.

\section{Les objets chez Bianca Zagolin}

Ayant compris les liens rattachant les objets au passé, la protagoniste bourgeoise d'Une femme à la fenêtre ne se sépare pas de ses affaires avec facilité : "Elle qui s'était toujours crue sans attaches matérielles ne supportait pas l'idée d'avoir à écarter la moindre parcelle de son passé " (p. 34). Limitée à trois malles et quelques bagages à main pour ses affaires et pour celles de ses trois filles, Aurore doit envoyer ses meubles, le piano, le berceau de la petite, la bibliothèque de son défunt mari et de nombreux autres objets personnels dans la maison ancestrale où ils rejoignent les autres objets abandonnés par les membres de la famille qui ont émigré. Le narrateur ou la narratrice hétérodiégétique de Zagolin attire l'attention d'une manière concise, à la fois toute classique et poétique sur l'importance des objets pour soutenir et la mémoire et notre conscience d'exister : "L'histoire s'érige ainsi sur ces objets qu'un temps d'allégresse a nimbés de lumière, les sauvant de l'oubli, et l'on ne peut s'en sevrer impunément car la mémoire y fonde la continuité de la conscience, sa seule certitude d'exister " (p. 35) Et si cette façon de concevoir l'importance des objets dans le temps est présente aussi dans la fiction de Ricci, la prise en charge du récit par un narrateur traditionnel, omniscient chez Zagolin laisse peu de place à l'interprétation du lecteur.

Aurore écarte des objets de première nécessité en faveur d'objets superflus, retenus pour leur beauté :

un service à thé venu de Chine, les rideaux de dentelle de la salle à manger et tous les couvre-lits de pure soie à ramages, une soupière ventrue peinte à la main $[\ldots]$. Aurore se ménageait ainsi des appuis, des points de repère issus de son passé pour que son être ne se liquéfiât pas entièrement dans la sombre épaisseur de l'inconnu. Et, pour ce faire, elle n'avait retenu, parmi les objets familiers, que ceux dont la beauté gratuite avait illuminé ses jours. (p. 36).

Ces objets feront partie de la nouvelle vie d'Aurore où " ils resteraient éternellement les signes tangibles d'un ailleurs où elle aurait jadis promené une âme distante mais soumise " (p. 37). Chez Zagolin, comme chez Ricci, les objets acquièrent le statut de signes. Et si Aurore cherche à s'imprégner de la beauté du paysage italien avant de le quitter, par contre, le seul être humain qu'elle regrettera est un vieux peintre qui avait rendu hommage à sa 
beauté en la peignant. Soumise aux règles traditionnelles de son milieu, Aurore trouve dans le départ sa revanche sur des gens qui lui ont miné la vie de contraintes. Quoiqu'elles soient diamétralement opposées, Aurore et Cristina, les protagonistes féminins de Zagolin et de Ricci, rêvent de trouver en Amérique la liberté dont on prive la femme en Italie.

Comme Vittorio, Aurore éprouve de la difficulté à accepter l'aspect irréel que prennent les lieux et les choses pendant son absence. Si la traversée vers l'Amérique donne à sa vie passée en Italie " la consistance d'un songe " (p. 49), son retour en Italie, presque deux ans plus tard, donne à sa vie montréalaise un aspect tout aussi irréel (p. 137). L'expérience d'Aurore de retour dans sa petite ville natale ressemble aussi à l'expérience de Vittorio à Valle del Sole et cela non seulement dans la mesure où " rien ne concord[e] avec le souvenir " (p. 143) mais aussi dans la réalisation que le passé ne pourra plus servir de refuge (p. 143).

Son déracinement s'était accompli sans appel car l'image mythifiée du pays jalousement conservée comme ultime recours, maintenant s'effritait. Les murs lézardés du souvenir, à l'ombre desquels elle s'était si souvent réfugiée, s'écroulaient dans un fracas poussiéreux, et Aurore s'éloignait à une vitesse vertigineuse. (Une femme à la fenêtre, 153-154)

Cette réalisation explique sans doute le nouveau sentiment de détachement d'Aurore pour les choses qu'elle a eu jadis tant de mal à abandonner en Italie. Ayant compris que " le temps des choix, des déchirements était révolu " (p. 153), Aurore ne prend, un peu au hasard, que deux jardinières et quelques livres appartenant à la petite Adalie. Elle a hâte de partir, de retrouver sa vie à Montréal.

Pour la deuxième fois en l'espace de quelques mois, une réalité familière avait brusquement sombré à la ligne d'horizon. Les sites de ses promenades, les chambres où elle s'était reposée, tous les lieux qui s'étaient ouverts pour la recevoir se refermaient derrière elle; dénués de mémoire, ils n'étaient témoins que du présent, des habitués ou des derniers arrivés qui remplaçaient toujours les disparus, indifférents aux vicissitudes des vies qu'ils enfermaient un bref instant à l'intérieur de leurs limites. Et, tandis qu'Aurore se glissait dans son nouvel espace et essayait d'y reprendre pied sans trop se faire remarquer, le monde là-bas continuait étrangement à exister sans elle. Aurore lui en voulait pour sa parfaite et cruelle impassibilité. Il lui tardait de réaffirmer sa présence dans son ancien décor où ne s'étaient pas encore effacées les traces de son passage. (Une femme à la fenêtre,163-164)

Dans ce contexte, on comprend plus facilement son mécontentement quand, rentrée chez elle à Montréal, Aurore découvre qu'Adalie a réaménagé le salon pour lui faire une surprise (p. 164). 
Dans Les Nomades, les objets qui entourent Philippe et les siens parmi lesquels un service à thé en or massif, une tapisserie pastorale, des lustres - ne cessent d'évoquer leur appartenance à la noblesse, à une classe privilégiée, et cela nonobstant la diminution de leur patrimoine et un destin voué désormais à l'errance comme à l'angoisse du nomade. Après chaque fuite, après chaque déménagement, la mère et la grand-mère de Philippe, Elisabeth-Marie et Clara, se consacrent corps et âme au culte du passé et à la reproduction fidèle de leur espace vital.

Elles avaient depuis longtemps gravé dans leur mémoire la disposition de chaque meuble, bibelot, ornement et accessoire en un plan immuable qui devait garantir la continuité. Au long de leurs pérégrinations, sur d'innombrables scènes, du bangalow des colonies à l'élégant flat de Londres, de la villa italienne aux balustres de marbre au pavillon de campagne, aucune modification d'importance n'était venue interrompre la perpétuation de leur mode de vie. Appauvrissement, exil, humiliations, rien de tout ce qui s'était acharné sur elles n'avait empêché Clara et sa fille de s'asseoir, la taille bien droite, sur leur canapé à roses d'or et de se délecter de quelque gourmandise en promenant un regard plein d'aise sur tout ce luxe bien conservé. La fidélité mobilisait leurs ressources et leurs énergies, mais l'adaptation se faisait toujours dans leur sens, et c'est avec la plus grande désinvolture qu'elles coulaient le monde dans le moule familial. (Les Nomades, 58)

Ainsi, au début des années 60, après vingt-cinq ans de migrations, les duchesses achètent une maison à Vancouver selon les dimensions nécessaires pour la disposition habituelle de leurs meubles et de toutes les possessions matérielles dont elles aiment s'entourer. Elles cherchent ainsi à recréer un milieu familier qui leur sert à la fois de miroir, leur renvoyant leur image, et de paravent contre l'étrangeté effrayante du monde extérieur. Quels que soient les stratagèmes adoptés pour faciliter leur expérience de l'exil, il est évident que les duchesses ne sont pas tout à fait chez elles et ce constat se répercute au niveau du décor :

Le sort les avait condamnées à une vie désormais sans envergure, à cet espace étriqué où l'on avait dû grouper les fauteuils Louis XV autour d'une table à thé, au lieu de les disposer entre les portes-fenêtres d'une spacieuse galerie. Le noble ameublement, qui dans ses courbes enfermait la durée des siècles, avait échoué entre les murs parfaitement lisses du présent. Ici, les bronzes des commodes, la marqueterie et les lustres de Venise juraient avec les plafonds trop bas et les fenêtres panoramiques. Dans ce décor de la dissonance, l'exil des choses répondait à celui de l'âme. (p. 73) 
Ces femmes s'attendent toutefois à ce que Philippe continue dans cette tradition et qu'il joue pleinement son rôle dans la préservation du passé. Mais le jeune homme ressent son propre besoin de fuite et se révolte contre leurs nostalgies. Ainsi il est attentif aux paroles de Mme McClaughry, la diseuse de bonne aventure qui l'encourage à quitter Vancouver, à traverser le pays pour rompre avec ceux qui l'emprisonnent. Mais à Montréal, ses relations avec Adalie révèlent qu'il reste attaché, fidèle, malgré sa révolte, à l'éducation reçue. Le Vieux Monde représenté par les duchesses se fissure sous le regard d'Adalie qui défie les traditions et s'exprime ouvertement. Passionnée, indépendante et libre, Adalie incarne tout ce que sa mère Aurore aurait voulu être. De plus, elle symbolise l'intégration de la nouvelle génération réalisée grâce aux sacrifices de la première.

Quand Philippe et Adalie se quittent, rien n'empêche Elisabeth-Marie de reprendre son fils. À la mort de Clara, Elisabeth-Marie achète une propriété près de Montréal et reçoit fièrement Philippe dans "le décor des ancêtres» (p. 206) :

[...] elle le reçut parmi les antiquités, fière d'avoir achevé son œuvre de conservation. En temps et lieu, elle léguerait à Philippe les biens de la famille, et aucune étrangère ne viendrait en réclamer sa part. Maintenant qu'il avait dépassé la trentaine et renié les rébellions et la verdeur de ses vingt ans, Philippe savourait, autant qu’avait pu le faire Clara à son apogée, son appartenance au passé dont il prendrait la charge à son tour en héritier légitime. (p. 207)

Mais si Philippe semble succomber à l'appel des ancêtres, il le fait tout en sachant que c'est pour les perdre et se perdre de façon définitive :

C'est à cela qu'aboutissait cette quête merveilleuse qu'il avait jadis entreprise en héros frondeur. A ce vide parfumé, à une existence de son invention. Le poids de l'héritage aurait eu raison de lui aussi; il s'accomplissait en sa personne, non pour l'enrichir, mais pour l'effacer, l'abîmer à son tour dans le secret ou l'oubli. Il n'y aurait pas de suite. Philippe ne laisserait derrière lui ni semence, ni souvenirs, ni tendresses meurtries. Seulement le silence, la mémoire étouffée à jamais. (p. 208)

Ainsi au suicide physique d'Aurore dans Une femme à la fenêtre répond le suicide métaphorique de Philippe qui se soumet finalement à l'impératif ancestral dans Les Nomades. L'un et l'autre, cependant, sont révélateurs de l'impossibilité de bien s'intégrer dans la vie, quel que soit le lieu de son déroulement, si on ne sait pas gérer ses attaches au passé, aux objets qui l'évoquent, et se détacher des gens qui nous y retiennent. Ainsi c'est Adalie, 
orpheline de père et de mère, qui montre le chemin à suivre aux enfants de l'immigration.

Quant à Vittorio, cependant, lui aussi orphelin de mère et de père, il cherche une voie toute personnelle dans l'écriture. Contrairement à Philippe qui se laissera étouffer par l'univers maternel et ancestral, Vittorio trouvera peut-être dans l'écriture le moyen de 'racheter' le passé en acceptant à la fois sa pluralité (car chacun retire une expérience différente d'un même événement) et l'impossibilité de le représenter dans son intégralité en passant par la mémoire, les mots et les objets. Témoins d'un autre monde et ennoblis d'un sens qui les dépasse, les objets ne peuvent combler chez l'immigré ni ses sentiments de perte et d'aliénation ni son besoin de retenir (pour ne pas se perdre lui-même) un monde révolu et insaisissable.

\section{University of Waterloo}

\section{Bibliographie d'ouvrages consultés}

Alfano, Michelle. "Saints and the Hereafter: An Interview with Nino Ricci." VIA: Voices in Italian Americana 6 (1995):11-31.

Baena, Rosalía. "Italian-Canadian Double Perspective in a Childhood Narrative: Nino Ricci's Lives of the Saints" in Tricks with a Glass: Writing Ethnicity in Canada, ed. Rocì G. Davis and Rosalìa Baena. Amsterdam: Rodopi, 2000, pp. 93-109.

Bernier, Silvie. "Récits d'Exil: Fictions d'Identité" in Palinsesti Culturali: Gli Apporti delle Immigrazioni alla Letteratura del Canada, eds. Anna Pia De Luca, Jean Paul Dufiet, Alessandra Ferraro. Udine: Forum, 1999, pp. 205-218.

Besemeres, Mary. "Different Languages, Different Emotions? Perspectives from Autobiographical Literature." Journal of Multilingual and Multicultural Development 25 (2004):140-158.

Camarca, Silvia. "Code-Switching and Textual Strategies in Nino Ricci's Trilogy." Semiotica: Journal of the International Association for Semiotic Studies / Revue de l'Association Internationale de Sémiotique 154:1-4 (2005): 225-241.

Canton, Jeffrey. "Recreating Paradise: Nino Ricci" in The Power to Bend Spoons: Interviews with Canadian Novelists, ed. Beverley Daurio. Toronto: Mercury, 1998, pp. 132-140.

Cro, Stelio. "Nino Ricci's Narrative and the Search for the Collective Unconscious of the Italian-American." Canadian Journal of Italian Studies 19 (1996):134149.

Dvorak, Marta. "Nino Ricci's Lives of the Saints: Walking Down both Sides of the Street at the Same Time." Commonwealth Essays and Studies 17 (1994): 58-66. Ireland, Susan. "Textualizing the Experience of Italian Women Immigrants." in Textualizing the Immigrant Experience, eds. Susan Ireland and Patrice J. Proulx. Westport: Praeger Publications, 2004, pp. 99-115

Leclaire, Jacques. "L'Exorcisme des racines dans la trilogie de Nino Ricci." Etudes 
Canadiennes / Canadian Studies: Revue Interdisciplinaire des Etudes Canadiennes en France 56 (2004): 155-169.

Niccoli, Gabriel. "Between Two Strange and Distant Shores: Fragments of Personal Connectedness to Nino Ricci's Lives of the Saints Trilogy." New Quarterly: Canadian Writers and Writing 93 (2005): 131-142.

Ricci, Nino. Where She Has Gone. Toronto: McClelland \& Stewart, 1997.

Ricci, Nino. In a Glass House. Toronto: McClelland \& Stewart, 1993.

Ricci, Nino. Lives of the Saints. Dunvegan: Cormorant Books, 1990.

Rimmer, Mary. "Nino Ricci: A Big Canvas." Studies in Canadian Literature / Etudes en Littérature Canadienne 18 (1993): 168-184.

Said, Edward. Reflections on Exile and Other Essays. Cambridge: Harvard University Press, 2002.

Tell, Edmond. "Into the Dark: An Interview with Nino Ricci." New Quarterly: Canadian Writers and Writing 93 (2005): 101-129.

Von Flotow, Luise. "Translating Women of the Eighties: Eroticism, Anger, Ethnicity." Culture in Transit: Translating the Literature of Quebec, ed. Sherry Simon. Montreal: Véhicule Press, 1995, pp. 31-46.

Zagolin, Bianca. Les Nomades. Montréal: Editions de l'Hexagone, 2001.

Zagolin, Bianca. "Le métissage culturel et littéraire: une réflexion personnelle" in Palinsesti Culturali: Gli Apporti delle Immigrazioni alla Letteratura del Canada, eds. Anna Pia De Luca, Jean Paul Dufiet, Alessandra Ferraro. Udine: Forum, 1999:19-26.

Zagolin, Bianca. Une Femme à la fenêtre. Paris: Éditions Robert Laffont, 1988. Zucchero, Jim. "What's Immigration Got to Do With It? Postcolonialism and Shifting Notions of Exile in Nino Ricci's Italian Canadians" in Is Canada Postcolonial? Unsettling Canadian Literature, ed. Laura Moss. Waterloo: Wilfrid Laurier University Press, 2003, pp. 252-267. 